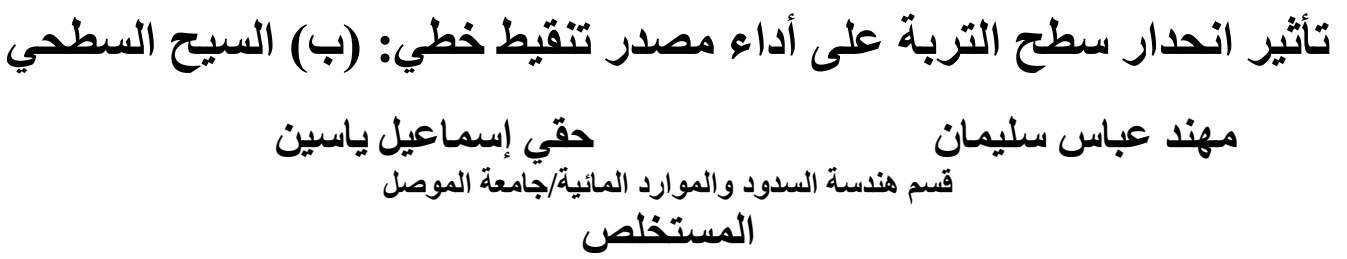

تهدف الاراسة إلى معرفة التأثير المتداخل لكل من انحدار سطح التربة و معدل إضـافة المـاء والرطوبـة الابتدائية

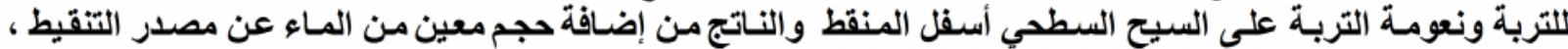

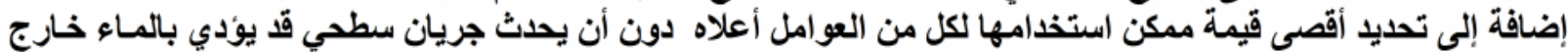

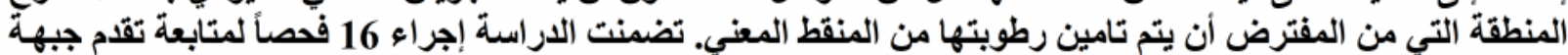

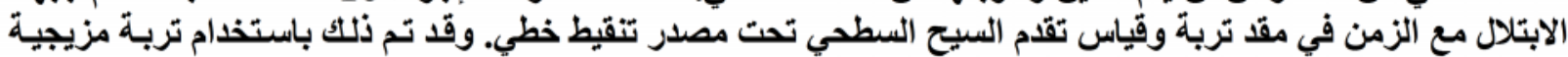

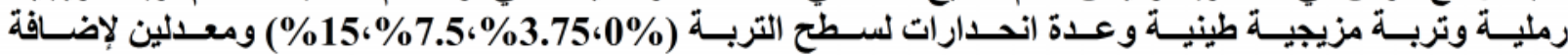

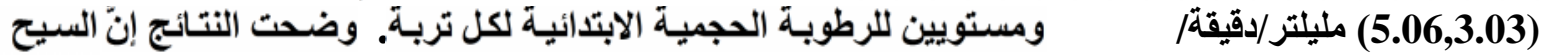

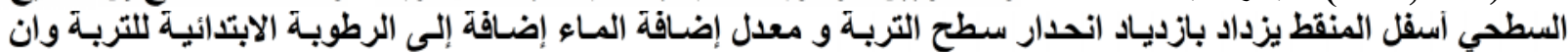

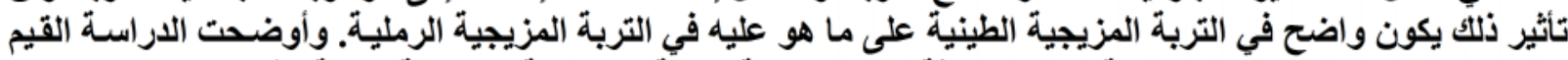

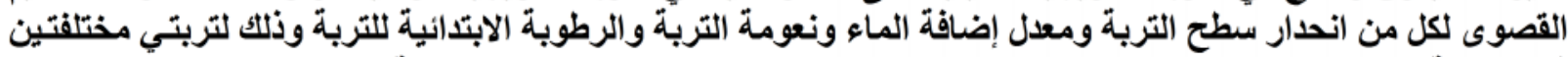
في النسجة عند استخدام مصدر تنقيط خطي بحيث لا يحدث جريـان سطحي خـارج المنطقة التي يتم تـامين رطويتها من

\title{
Effect Of Soil Surface Slope On The Performance Of Trickle Line Source:(B)Surface Runoff
}

\begin{abstract}
The present study aims to find out the einterferences effects of the soil surface slope, the water application rate, soil type, and the initial soil moisture on the local surface runoff, which is resulting from the application of certain water volume by trickle line source. In addition to determining the maximum applicable value for each of the above mentioned factors, which can be used without the surface runoff occurs which may be lead the water out of the area that is supposed to be secure the moisture from concerned trickler. The study included 16 test to monitor the advance of the wetting front and the local surface runoff with time in the soil profile under trickle line source. This was done by using sandy loam soil and clay loam soil, several soil surface slopes $(0 \%, 3.75 \%, 7.5 \%, 15 \%)$, two water application rates $(3.03,5.06) \mathrm{ml} / \mathrm{min} / \mathrm{cm}$ and two levels of the volumetric initial moisture for each soil. The study results showed that the surface runoff downstream trickler increases with the increase of the soil surface slope or the increase the water application rate or the increase of the initial soil moisture and the surface runoff effects are greater in the clay loam soil than the sandy loam soil. And study proposed the maximum value for each of the soil surface slope, the water application rate, and the volumetric initial soil moisture for study's soils when using trickle line source, so that the surface runoff does not happen outside the area that is supposed to be secure moisture from the concerned trickler.
\end{abstract}




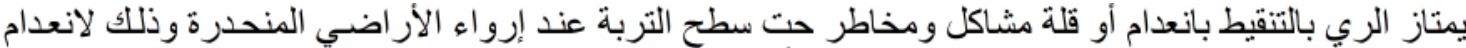

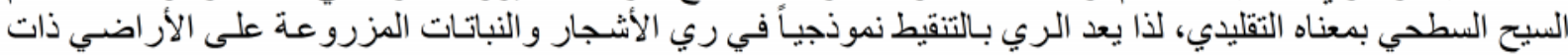

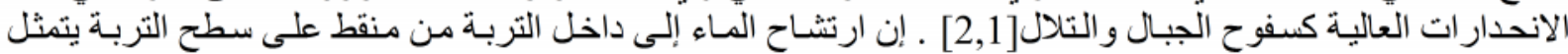

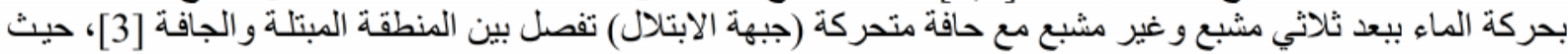

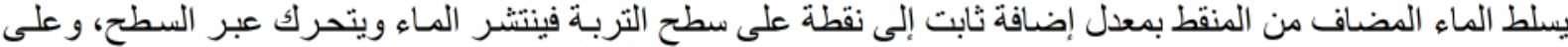

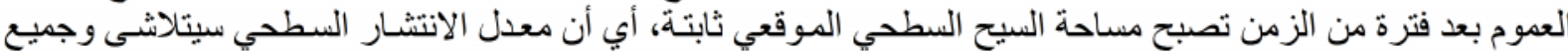

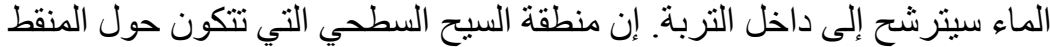

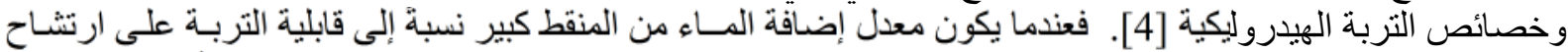

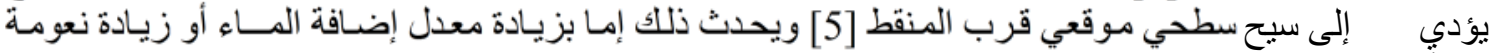

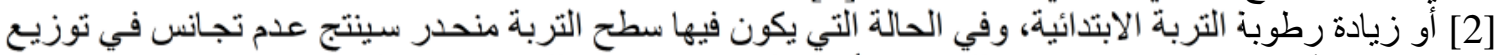

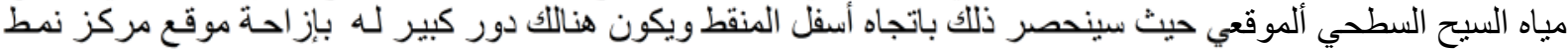

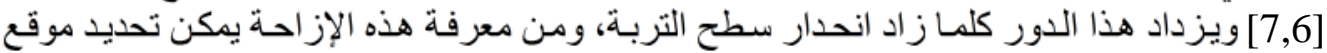

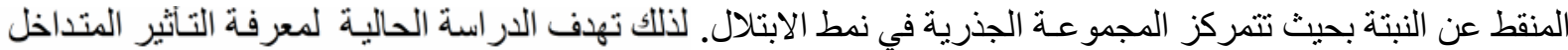

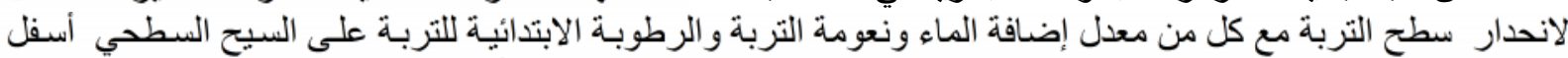

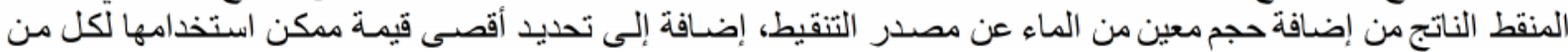

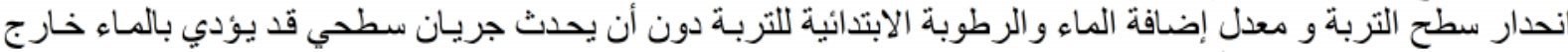
لمنطقة التي من المفترض أن ينت تامين رطوبتهاء ون المناف المنط المعني.

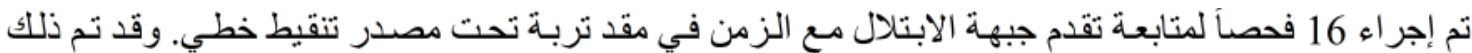

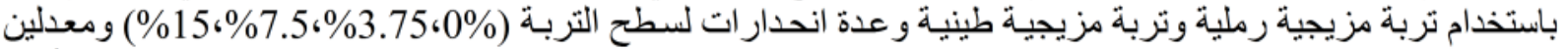
54

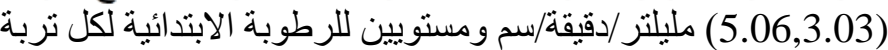

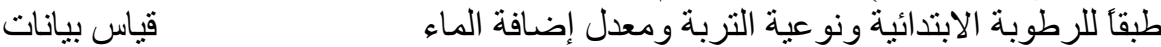

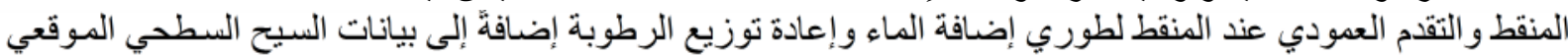

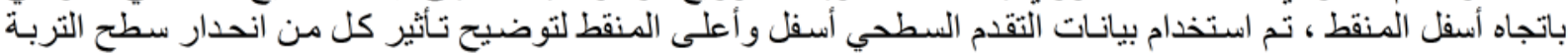

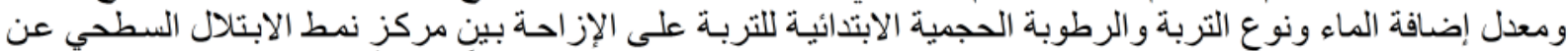

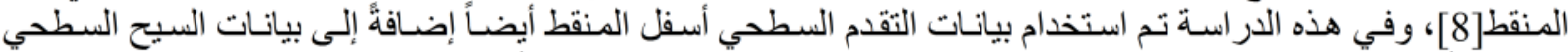

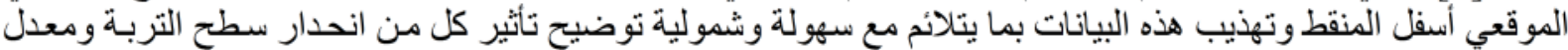

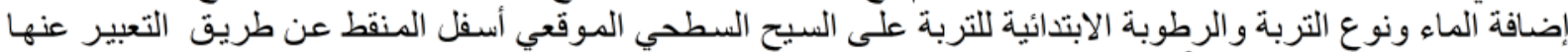

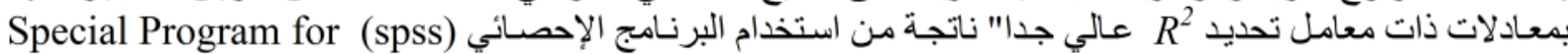

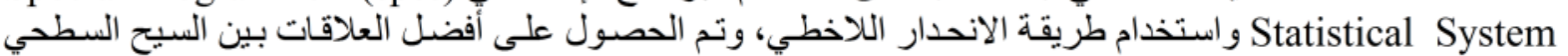

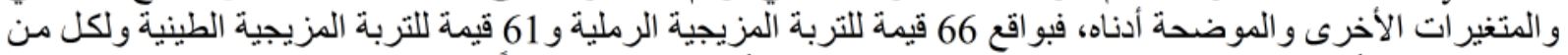

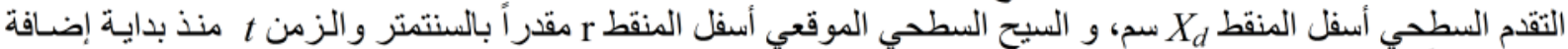
الماء مقدراً بالذقيقة و الرطوبة الابتدائيسة للتربة

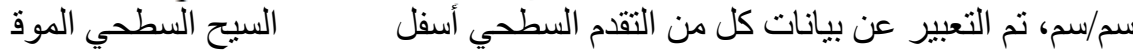

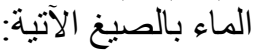

$$
\begin{gathered}
X_{d=}=A_{1}{ }^{*} t^{A 2} * \theta_{i}{ }^{A 3} * q^{A 4}+A_{5} * t^{A 6} * S^{A 7} \\
r=A_{1} * t^{A 2} * q^{A 3}\left[A_{4} * \theta_{i}{ }^{A 5}+A_{6} * S^{A 7}\right]
\end{gathered}
$$

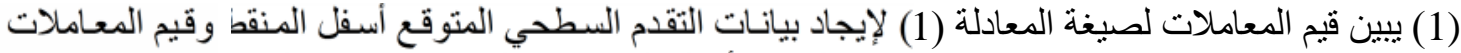

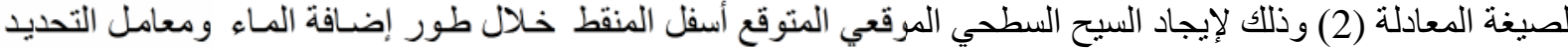


لتربتي الدر اسـة. وقد تم إجر اء اختبـار ات إحصـائية (One-Way ANOVA و T.test) باستخدام البرنـامج الإحصـائي

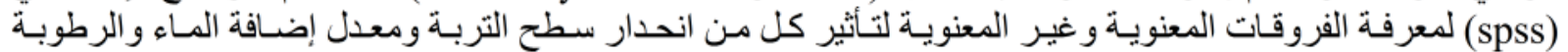
السيح السطحي الموقعي. الابتدائية للتربة

(1): قيم المعاملات لصيغة المعادلة (1) للتعبير عن بيانات التقدم السطحي أسفل المنقط ومعامل التحديد لتربتي

\begin{tabular}{|c|c|c|c|c|c|c|c|c|c|}
\hline$R^{2}$ & $A_{7}$ & $A_{6}$ & $A_{5}$ & $A_{4}$ & $A_{3}$ & $A_{2}$ & $\overline{A_{1}}$ & المتنغرات & \\
\hline 0.989 & 0.35 & 0.4708 & 4.6082 & 2.0574 & 0.3197 & 0.4708 & 0.2886 & $X_{d}$ & مزيجية رملية \\
\hline 0.991 & 0.35 & 0.6298 & 2.3368 & 1.2493 & 0.1356 & 0.6298 & 0.5058 & $X_{d}$ & مزيجية طينية \\
\hline 0.972 & 0.6 & 0.4136 & 1.6837 & 0.8547 & 2.1685 & 0.6261 & 0.5082 & $r$ & مزيجية رملية \\
\hline 0.985 & 0.6 & 0.251 & 1.1984 & 0.491 & 2.0515 & 0.748 & 0.628 & $r$ & مزيجية طينية \\
\hline
\end{tabular}

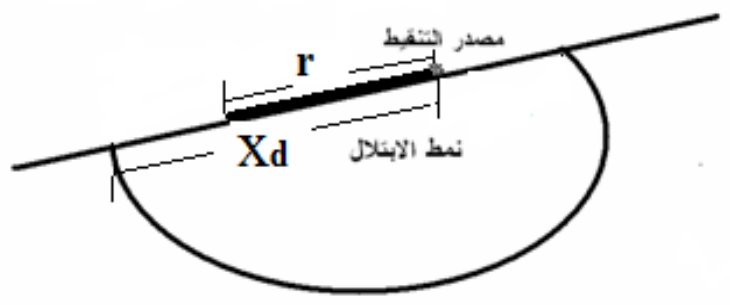

$\underline{(r)}$ السيح السطىى

$\left(\mathrm{X}_{\mathrm{d}}\right)$

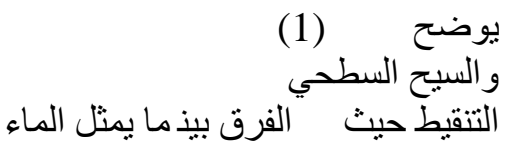

(r)

الشكل(1):التقدم السطحي أسفل المنقط والسيح السطحي ألموقعي

تأثير انحدار سطح التربـة: يوضـح الثـكلان (2 و3)

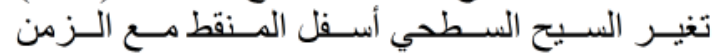

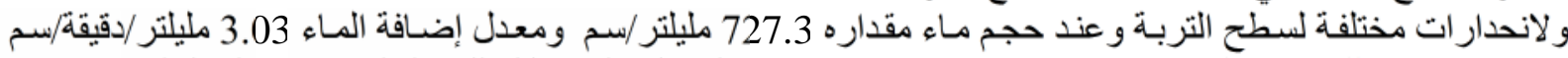

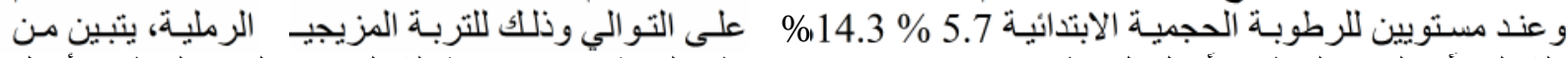

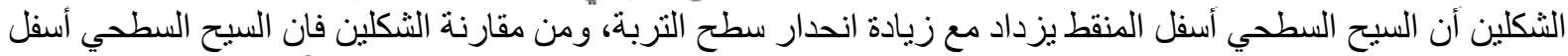

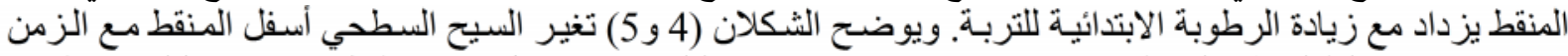

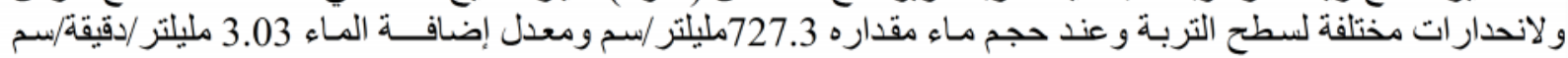

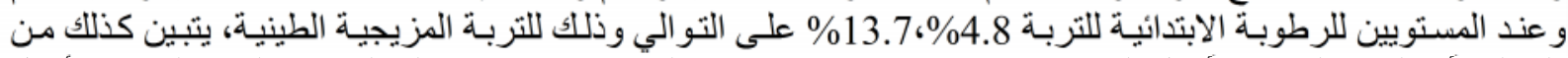

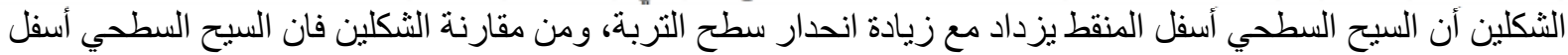

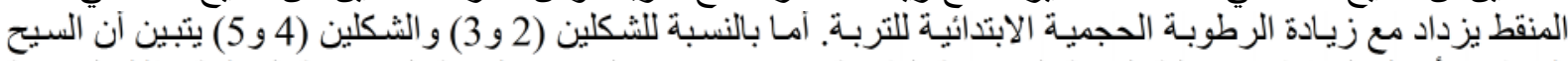

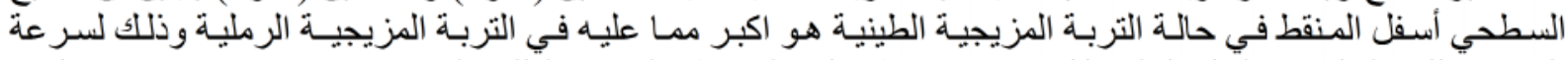

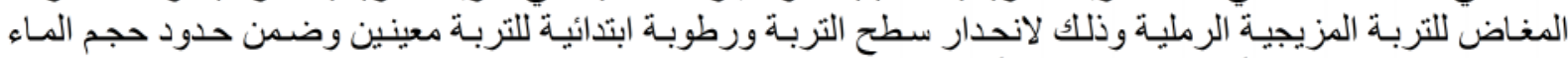

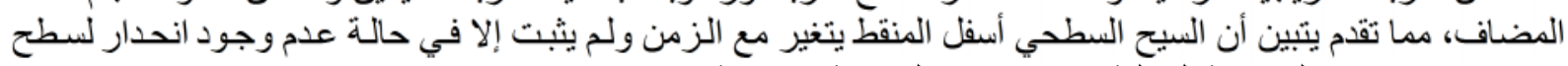

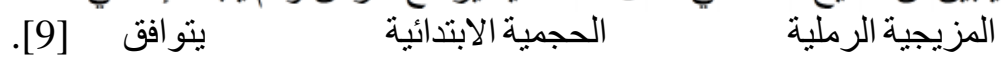

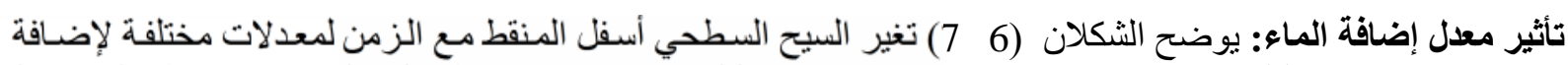

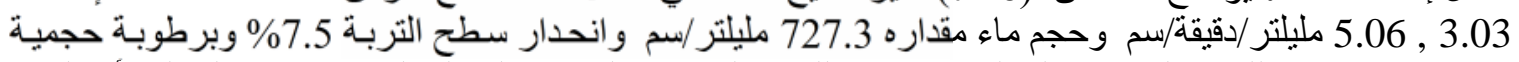

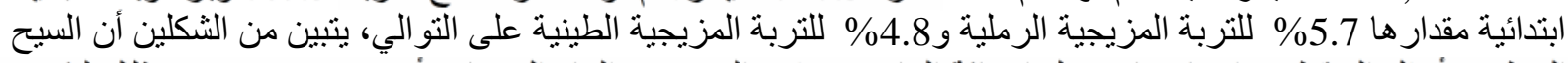

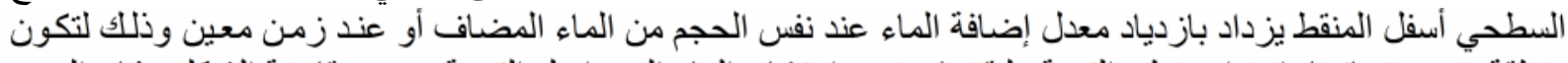

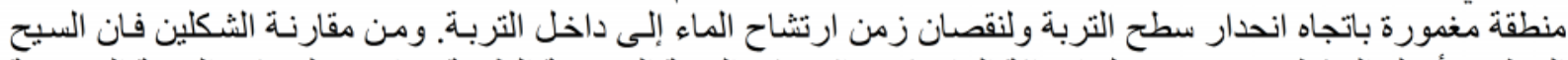

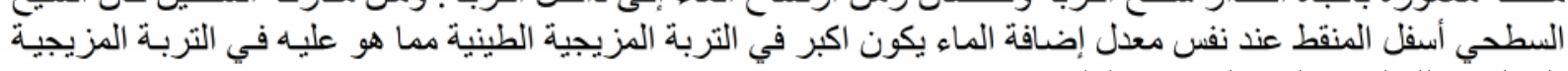
الرملية وذللك لحجم الماء المضاف الكنلي.

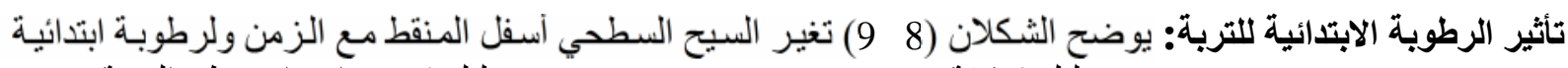
727.3 مليلتر/سم وانحدار سطح التربة النية 72.5 3.03 مليلتر /دقيقة/ 


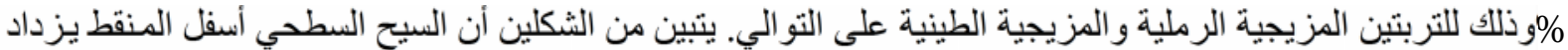

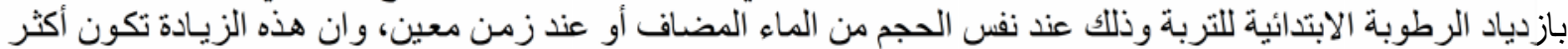

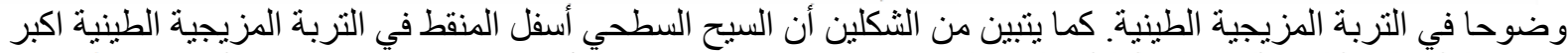

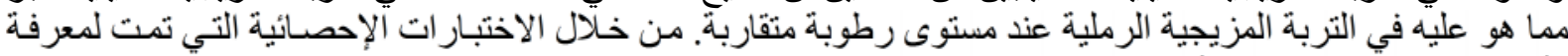

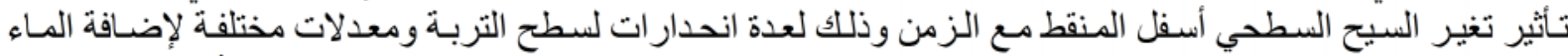

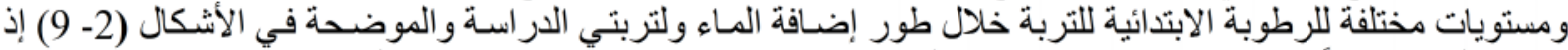

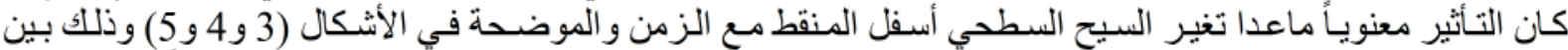

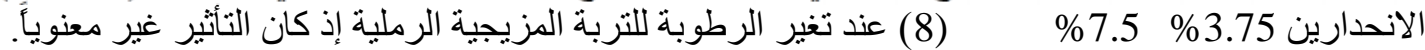

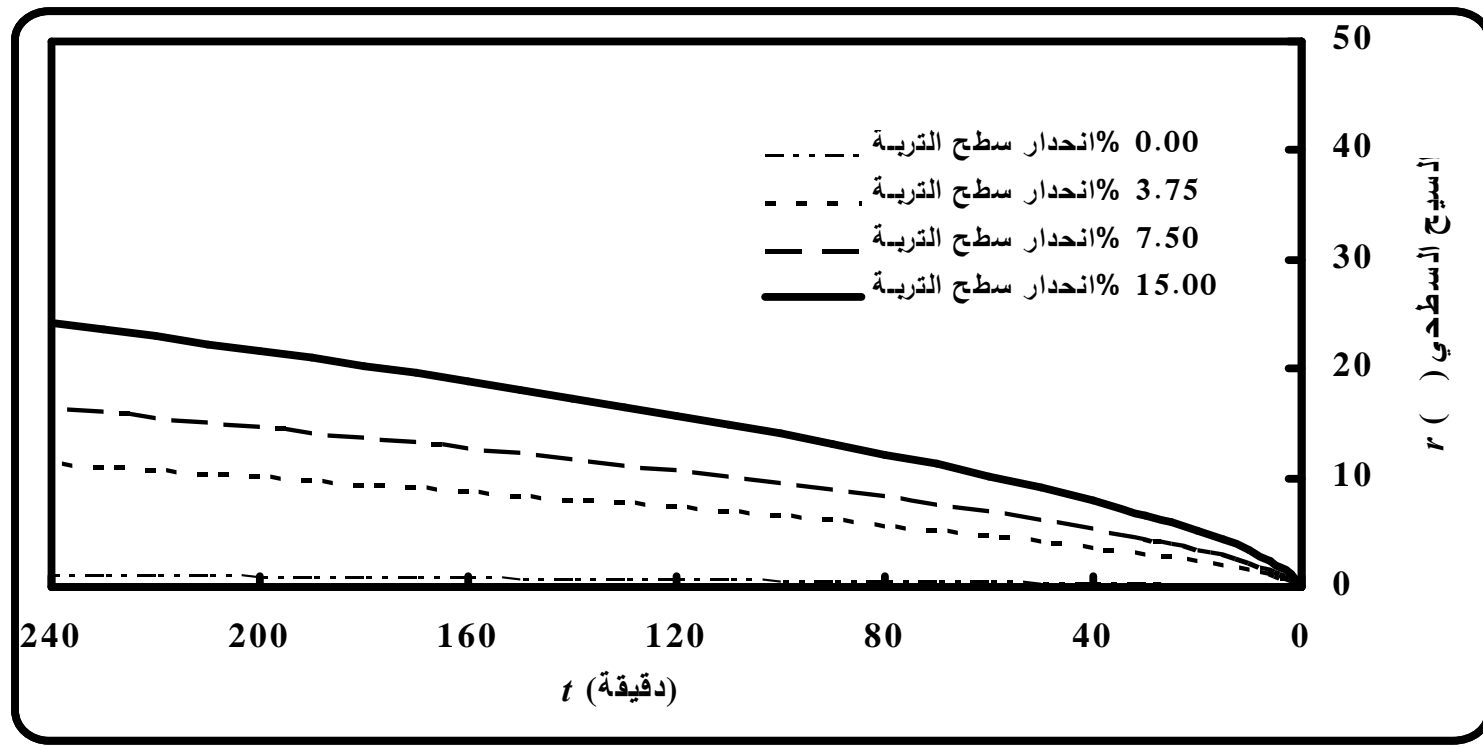

ابتدائية

3.03 مليلتر/دقيقة/سم

727.3

(2)

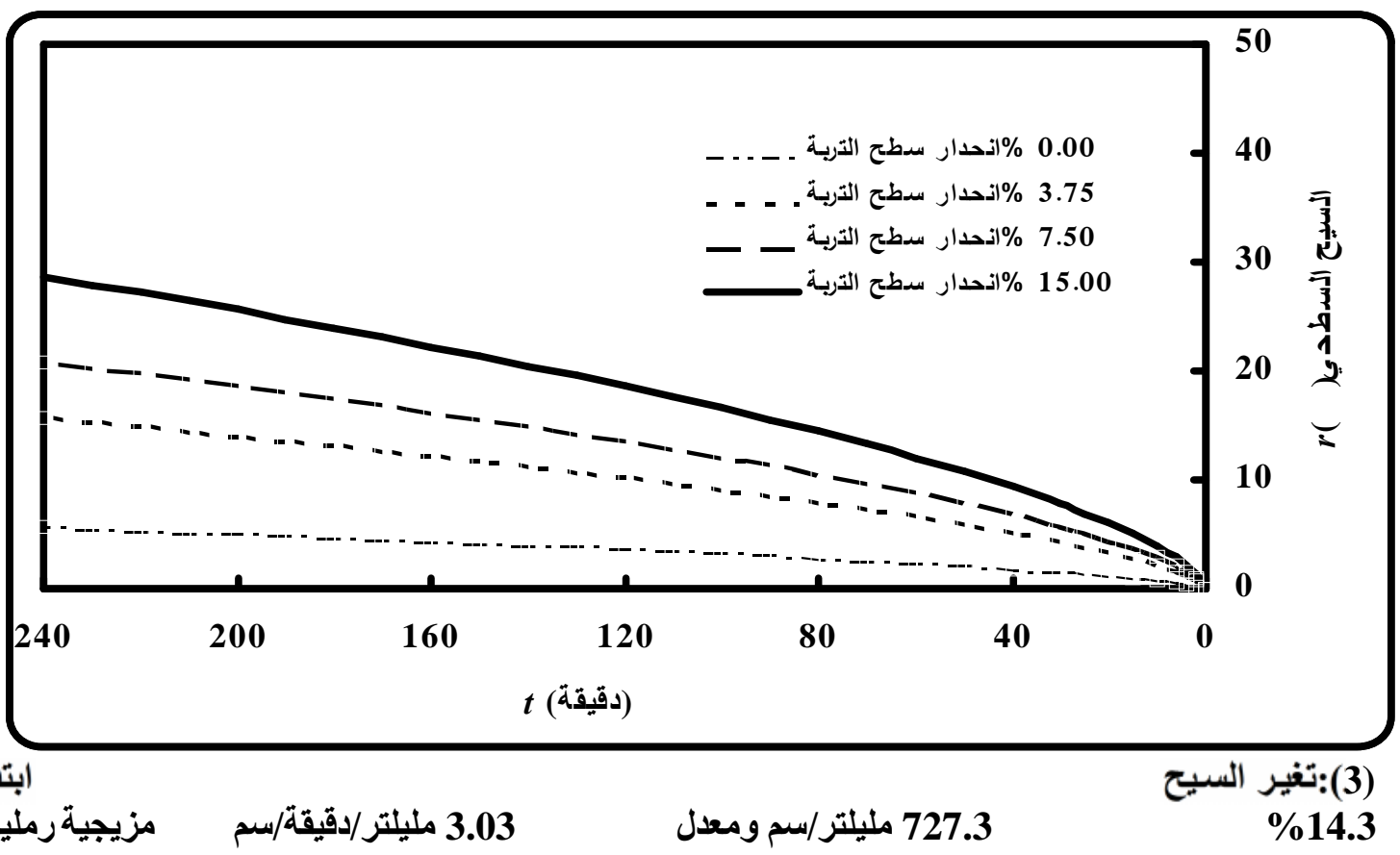


ياسين: تأثير انحدار سطح التربة على أداء مصدر تنقيط خطي: (ب) السيح السطحي

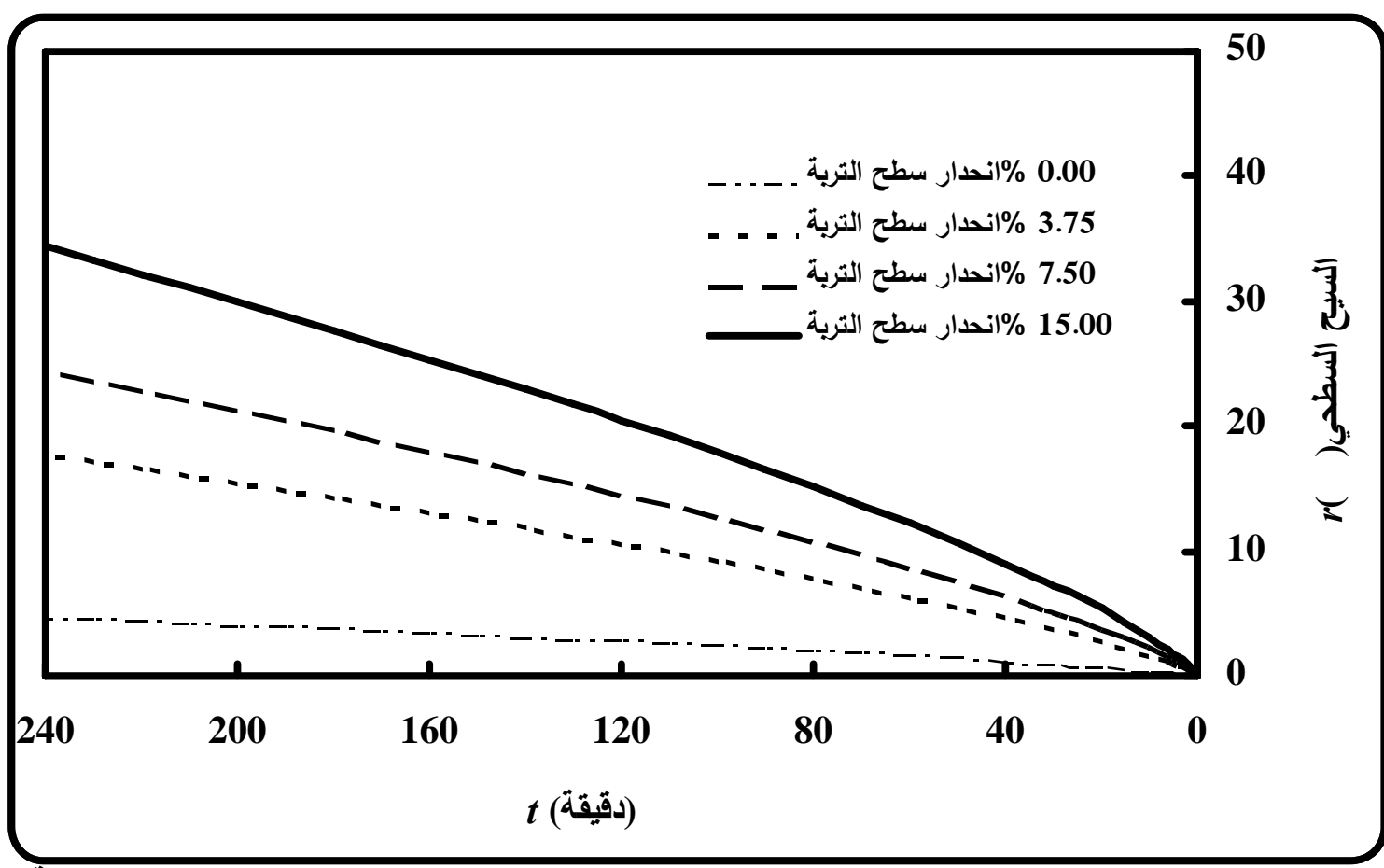

ابتدائية

3.03 مليلتر/دقيقة/سم مزيجيةطينية.

727.3 مليلتر/سم ومعدل

(4):تغير السيح:(4)

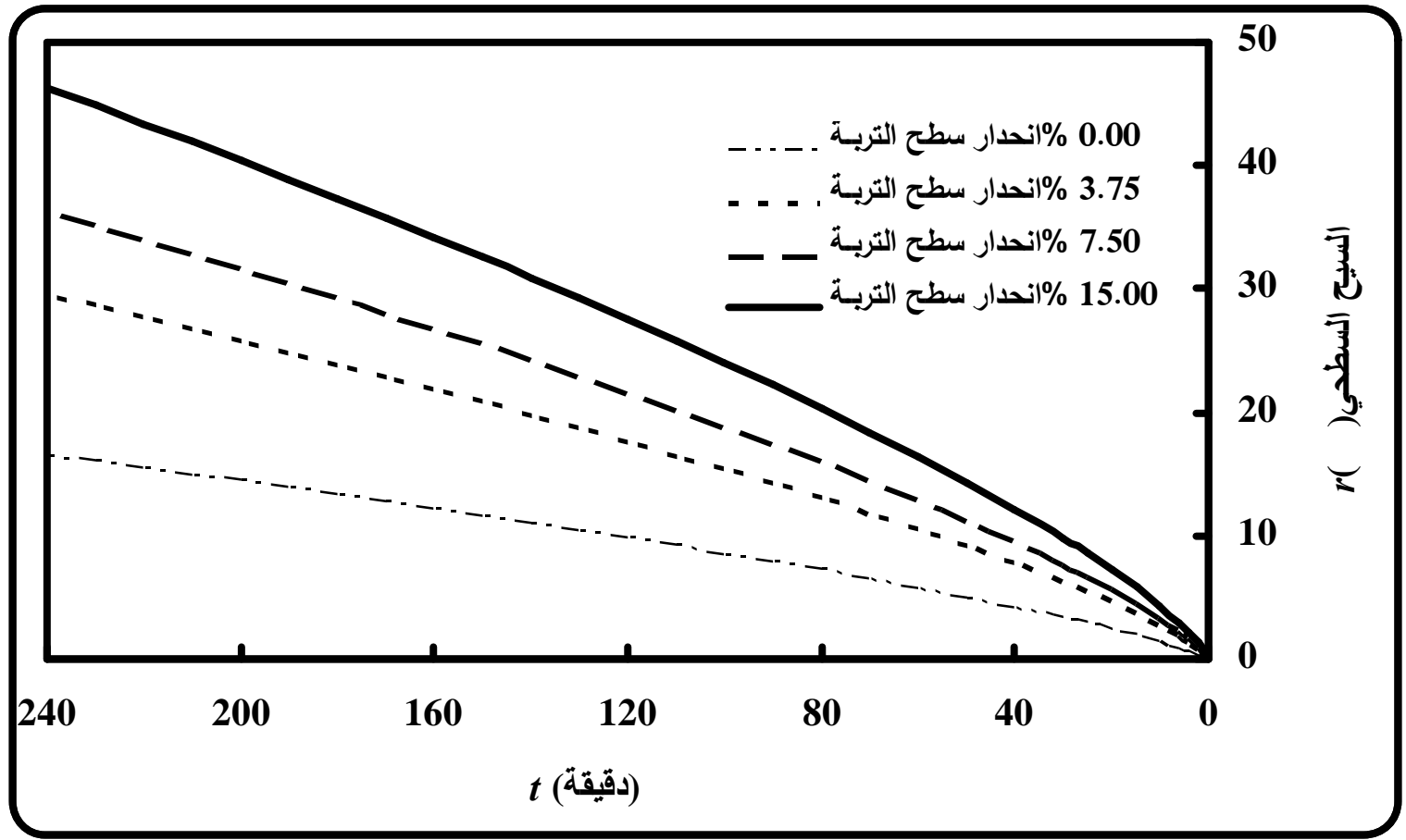

ابتدائية

727.3 مليتز/سم ومعدل

(5):تغير السيح:(5)

$\% 13.7$ 
No. 5

Dec. 2014
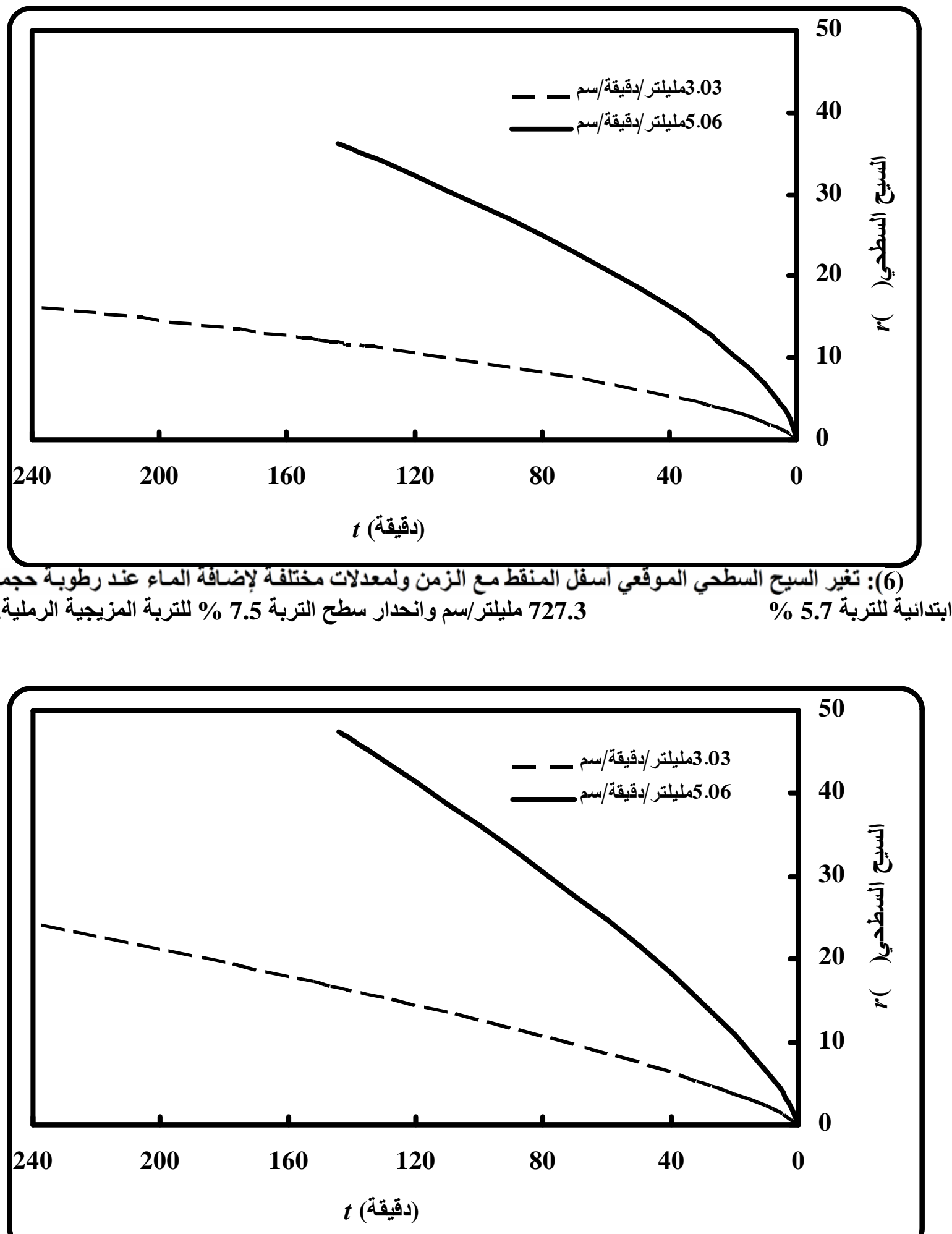

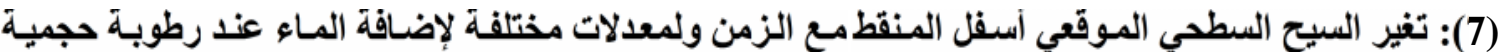
ابتدائية للتربة 4.8 \% \% 727.3 مليلتر/سم وانحدار سطح التربة 7.5 \% للتربة المزيجية الطينية. 
ياسين: تأثير انحدار سطح التربة على أداء مصدر تنقيط خطي: (ب) السيح السطحي

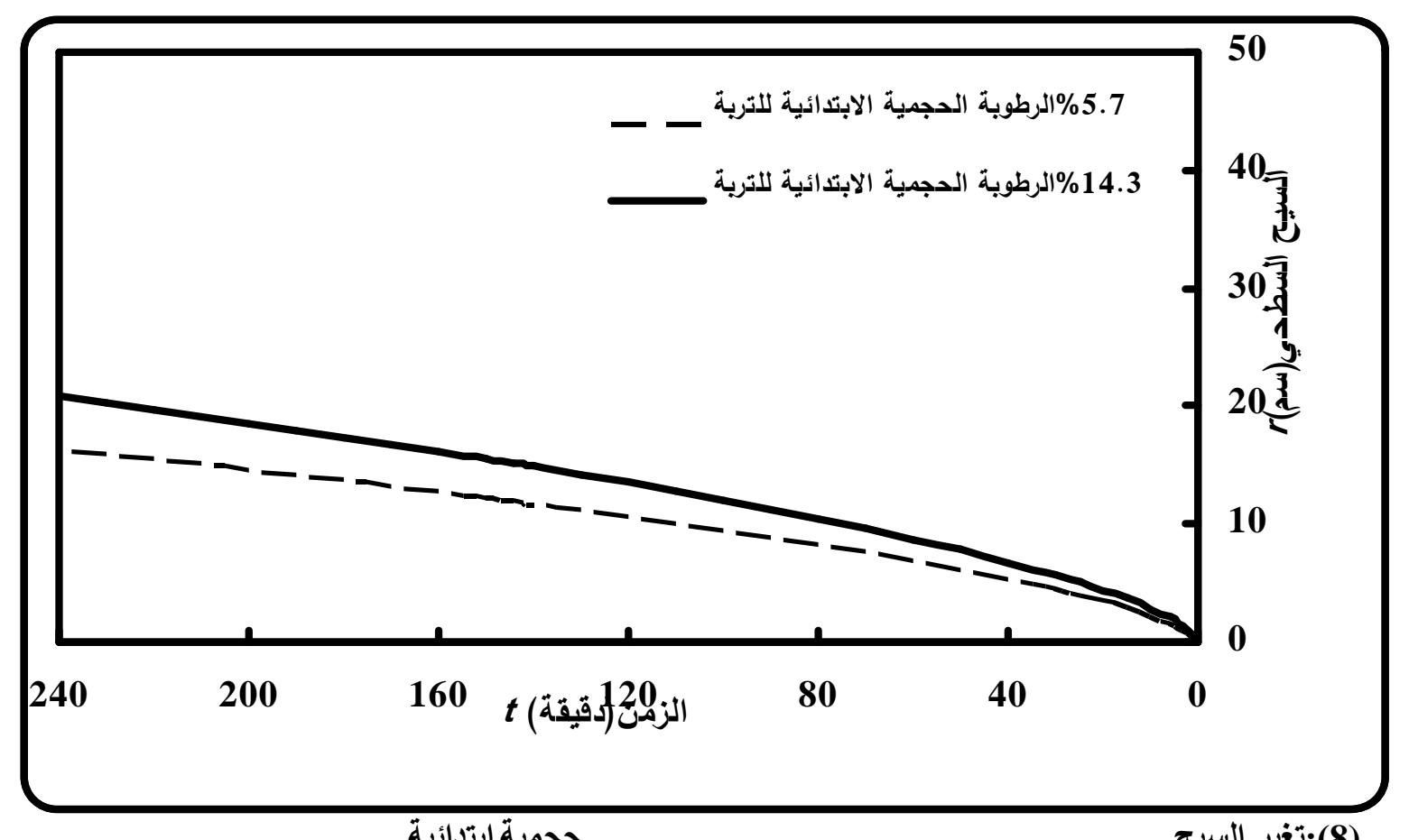

3.03 مليلتر/دقيقة/سم وحجم الماء المضاف 727.3 مليلتر/سم وانحدار سطح التربة 7.5 \% لتربة مزيجية رملية.

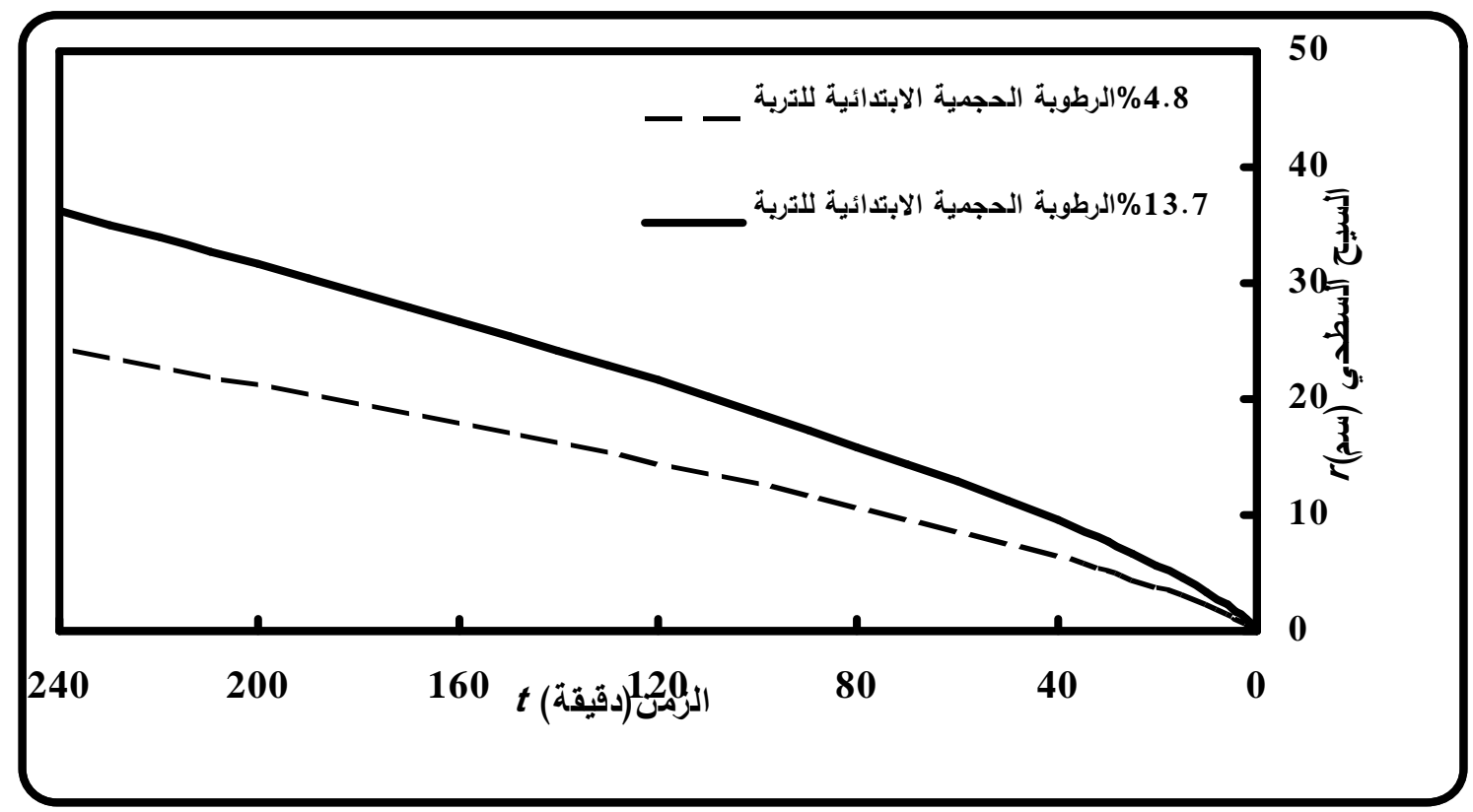

حجمية ابتدائية

\% 7.5 \% لتربة مزيجية طينية. 727.3 مليلتز/سم وانحدار

(9):تغير السيح 3.03 مليلتر/دقيقة/سم وحجم الماء

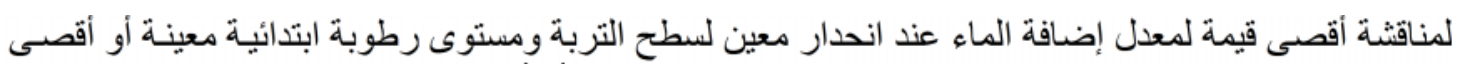
انحدار لسطح التربة عند معدل إضافة ماء معين ومستوى رطوبة ابتدائية معينة أو أقصى رطوبة التصنية رطية ائية عند معدل إضـافة 
ماء معين و انحدار لسطح التربة معين، فلتحديد أقصى قيمة ممكن استخدامها دون أن يحدث جريان سطدي قد يؤدي بالمباء

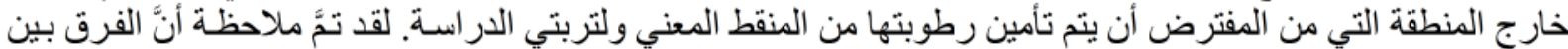

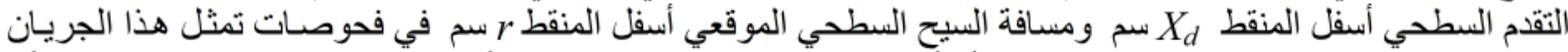

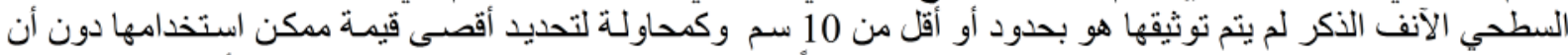

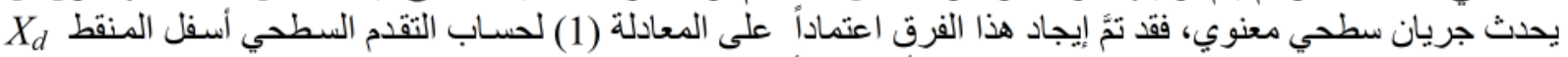

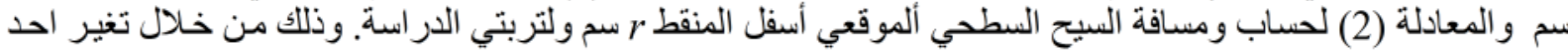

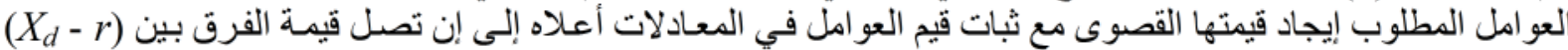

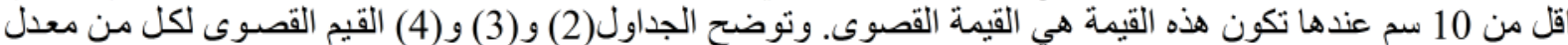

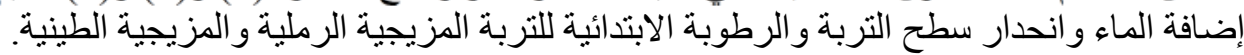

عند مستو يين للرطوبة الابتدائية للتربة و انحدار ات مختلفة لسطح مختلفة للتربة

المزيجية الرملية والمزيجية الطينية.

\begin{tabular}{|c|c|c|c|c|c|}
\hline \multicolumn{6}{|c|}{ أقصى معدل لإضافة الماء مليلتر/دقيقة/سم } \\
\hline \multicolumn{3}{|c|}{ مزيجية طينية } & \multicolumn{3}{|c|}{ مزيجية رملية } \\
\hline \multicolumn{3}{|c|}{ الرطوبة الابتدائية للتربة 10\% } & \multicolumn{3}{|c|}{ الرطوبة الابتدائية للتربة 10\% } \\
\hline$\% 20$ & $\% 15$ & $\% 10$ & $\% 20$ & $\% 15$ & $\% 10$ \\
\hline 4.7 & 5.1 & 5.8 & 4.1 & 4.55 & 6 \\
\hline \multicolumn{3}{|c|}{ الرطوبة الابتائية للتربة 20\% } & \multicolumn{3}{|c|}{ الرطوبة الابتدائية للتربة 20\% } \\
\hline$\% 20$ & $\% 15$ & $\% 10$ & $\% 20$ & $\% 15$ & $\% 10$ \\
\hline 3.9 & 4.1 & 4.35 & 3.7 & 4.05 & 4.8 \\
\hline
\end{tabular}

ر رلسطح التربة عند مستويين للرطوبة الابتدائية للتربة ومعدلات مختلفة لإضافة الماء وللتربتين

المزيجية الرملية و المزيجية الطينية.

\begin{tabular}{|c|c|c|c|c|c|}
\hline \multicolumn{6}{|c|}{$\%$} \\
\hline \multicolumn{3}{|c|}{ مزيجية طينية } & \multicolumn{3}{|c|}{ مزيجية رملية } \\
\hline \multicolumn{3}{|c|}{ الرطوبة الابتدائية للتربة 10\% } & \multicolumn{3}{|c|}{ الرطوبة الابتدائية للتربة 10\% } \\
\hline \multicolumn{3}{|c|}{ معدل لإضافة الماء مليلتر/دقيقة/سم } & \multicolumn{3}{|c|}{ لماء مليلتر/دقيقة/سم } \\
\hline 6 & 5 & 4 & 6 & 5 & 4 \\
\hline 9 & 16 & 36 & 10.5 & 12.5 & 21 \\
\hline \multicolumn{3}{|c|}{ الرطوبة الابتدائية للتربة 20\% } & \multicolumn{3}{|c|}{ الرطوبة الابتدائية للتربة $20 \%$} \\
\hline \multicolumn{3}{|c|}{ معدل لإضافة الماء مليلتر/دقيقة/سم } & \multicolumn{3}{|c|}{ معدل لإضافة الماء مليلتر/دقيقة/سم } \\
\hline 6 & 5 & 4 & 6 & 5 & 4 \\
\hline 0.9 & 4 & 16 & 7.5 & 9 & 15.5 \\
\hline
\end{tabular}


ياسين: تأثير انحدار سطح التربة على أداء مصدر تنقيط خطي: (ب) السيح السطحي

(4): أقصى رطوبة ابتدائية للتربة و لانحدار ات مختلفة لسطح التربة ومعدلات مختلفة لإضافة الماء للتربة المزيجية

الرملية و المزيجية الطينية.

\begin{tabular}{|c|c|c|c|c|c|}
\hline \multicolumn{6}{|c|}{ أقصى رطوبة ابتدائية للتربة \% } \\
\hline \multicolumn{3}{|c|}{ مزيجية طينية } & \multicolumn{3}{|c|}{ مزيجية رملية } \\
\hline \multicolumn{3}{|c|}{$\% 10$} & \multicolumn{3}{|c|}{$\% 10$} \\
\hline \multicolumn{3}{|c|}{ معدل لإضافة الماء مليلتر/دقيقة/سم } & \multicolumn{3}{|c|}{ ضافة الماء مليلتر/دقيقة/سم } \\
\hline 6 & 5 & 4 & 6 & 5 & 4 \\
\hline 8.8 & 15 & 23.5 & 13 & 19 & 25 \\
\hline \multicolumn{3}{|c|}{$\% 20$} & \multicolumn{3}{|c|}{$\% 20$} \\
\hline \multicolumn{3}{|c|}{ معدل لإضافة الماء مليلتر/دقيقة/سم } & \multicolumn{3}{|c|}{ معدل لإضافة الماء مليلتر/دقيقة/سم } \\
\hline 6 & 5 & 4 & 6 & 5 & 4 \\
\hline & 5.7 & 18 & & & 15 \\
\hline
\end{tabular}

1. السيح السطحي أسفل المنقط يتغير مع الزمن ولم يثبت إلا في حالة التربـة المزيجية الرملية و عند رطوبـة ابتدائيسة و اطئة ولسطح تربة مستوي وذللك لتأثير بمعدل غيض الكنّ ولثر من الثربة المزيجية الطينية.

2. إنَّ السيح السطحي أسفل المنقط يزداد بازدياد كل من انحدار سطح التربـة أو معدل إضـافة المـاء أو الرطوبـة الربـة الابتدائيسة

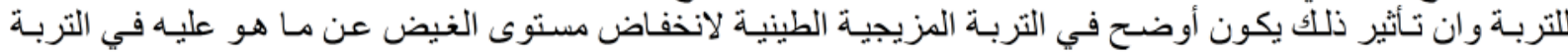
المزيجية الرملية.

3. قدمت الدر اسة أقصى قيمة لكل من انحدار سطح التربة ومعدل إضافة الماء ونعومة التربة والرطوبة الابتدائية للتربة

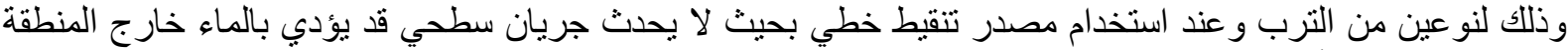
التي من المفترض أن يتم تامين رطوبتها من المنقط المعني.

1 حاجم، احمد يوسف و حقي إسماعيل ياسين " هندسـة نظم الري الحقلي" دار الكتب للطباعة و النشر، جامعة الموصل، .(1992) .

2. Merkley and Allen "Sprinkle and Trickle Irrigation Lectures ". Journal ,ASAE Transactions, International Committee on Irrigation and Drainage(ICID) Journal,and others.Utah stat University,Logan,Utah, (2007) Page 244.

3. Hammami,M.,H.Daghari, J. Balti, and M.Maalej "Approach for predicting the wetting front depth beneath a surface point source: Theory and numerical aspect" . Irrigation and Drainage (51), 2002 ,pp. 347-360.

4. Assouline, S. (2002) "The effect of microdrip and conventional drip irrigation on water distribution and uptake". Soil Sci. Soc. Am. J.(66) : 1630-1636.

5. Nath ,T. K .and Tiwar (2005) "Prediction of ideal wetting depth under point source - a numerical approach" IE (I) Journal.AG, Vol.(86). 
6. Lafolie, F., R.Guennelon, and M.Th. van Genuchten (1989) "Analysis of water flow under trickle irrigation:I.Theory and numerical solution”. Soil Sci. Soc. Am. J. (53):1310-1318

7.Zhimin Li and Logan W. APPERLEY(1992) "Water distribution patterns from trickle irrigation system". $11^{\text {th }}$ Australasian Fluid Mechanics Conference, Australia, p. 1287

لنو عين من التربة تحت مصدر تنقيط خطي ". الة ماجستير .(2013).

9. Youngs, E.G.,P.B.Leeds-Harrison and A.Alghusni (1999) "Surface ponding of coarsetextured soils under irrigation with a line of surface emitters". Journal of Agricultural Engineering Research (73) : 95-100.

تم اجراء البحث في كلية ألهندسة = جامعة ألموصل 